\title{
OPTIMAL SIGNALING AND DETECTOR DESIGN FOR POWER CONSTRAINED ON-OFF KEYING SYSTEMS IN NEYMAN-PEARSON FRAMEWORK
}

\author{
Berkan Dulek and Sinan Gezici \\ Department of Electrical and Electronics Engineering \\ Bilkent University, Bilkent, Ankara 06800, Turkey \\ \{dulek,gezici\}@ee.bilkent.edu.tr
}

\begin{abstract}
Optimal stochastic signaling and detector design are studied for power constrained on-off keying systems in the presence of additive multimodal channel noise under the NeymanPearson (NP) framework. The problem of jointly designing the signaling scheme and the decision rule is addressed in order to maximize the probability of detection without violating the constraints on the probability of false alarm and the average transmit power. Based on a theoretical analysis, it is shown that the optimal solution can be obtained by employing randomization between at most two signal values for the on-signal (symbol 1) and using the corresponding NP-type likelihood ratio test at the receiver. As a result, the optimal parameters can be computed over a significantly reduced optimization space instead of an infinite set of functions using global optimization techniques. Finally, a detection example is provided to illustrate how stochastic signaling can help improve detection performance over various optimal and suboptimal signaling schemes.
\end{abstract}

Index Terms - Stochastic signaling, on-off keying, Neyman-Pearson (NP) decision rule.

\section{INTRODUCTION AND MOTIVATION}

In power constrained binary communications systems, stochastic signaling; that is, modeling signals for transmitted symbols as random variables instead of deterministic quantities, can provide performance improvements in some scenarios $[1,2]$. This method has proven effective in reducing the average probability of error for power constrained communications systems over additive noise channels with multimodal probability density functions (PDFs). ${ }^{1}$

Although optimal signaling and detector design has been studied extensively in the literature according to various criteria (e.g., Bayes, minimax, Neyman-Pearson) when the noise is Gaussian, the noise can have significantly different probability distribution than the Gaussian distribution in some cases due to effects such as multiuser interference and jamming [4]. Joint optimization of signal structures and detectors in terms of error performance is investigated under an average power constraint in [2]. It is proven that the optimal performance can be achieved when the transmitted signal for each symbol is randomized between no more than two signal values and the corresponding maximum a posteriori probability (MAP) detector is employed at the receiver. In [5], optimal randomization among antipodal signal pairs and the corresponding MAP decision rules is studied under the assumption that the receiver knows which deterministic pair is transmitted. It is

${ }^{1}$ In [3], it is shown that if the channel has a continuously differentiable unimodal PDF with finite variance, the average probability of error versus average power is a nonincreasing convex curve when the transmitter employs antipodal signaling, and the receiver uses the maximum likelihood (ML) rule. In that case, stochastic signaling cannot improve the error performance. concluded that randomization between two detectors is sufficient to maximize the correct decision probability.

In detection theory, most of the research conducted on stochastic signaling has focused on improving the performance of detectors according to the average probability of error criterion $[1,2]$. Although the prior probabilities of the symbols are assumed to be equal in many communications systems, they can be unknown and nonequal in certain cases [6]. Furthermore, it may not be possible to impose cost structures on the decisions [7]. Under such scenarios, neither Bayesian nor minimax decision rules are applicable, and the Neyman-Pearson (NP) hypothesis testing provides a favorable alternative. For example, in wireless sensor network applications, a transmitter can send one bit of information (using on-off keying) about the presence of an event (e.g., fire), in which case the probabilities of detection and false alarm become the main performance metrics as in the NP approach. In this study, we consider a power constrained on-off keying communications system in the NP framework, and formulate the problem of jointly designing the signaling scheme and the decision rule (detector). Based on a theoretical analysis, it is shown that the optimal solution can be obtained by employing randomization between at most two signal values for the on-signal (symbol 1) and using the corresponding NP-type likelihood ratio test (LRT) at the receiver. Hence, the optimal parameters can be computed over a significantly reduced optimization space instead of an infinite set of functions. Finally, a detection example is provided to compare various optimal and suboptimal signaling schemes.

\section{OPTIMAL SIGNALING AND DETECTOR DESIGN}

Consider an on-off keying communications system, in which the receiver acquires $M$-dimensional observations over an additive noise channel and decides between the two hypotheses $H_{0}$ or $H_{1}$, which are modeled as

$$
H_{0}: \mathbf{Y}=\mathbf{N}, \quad H_{1}: \mathbf{Y}=\mathbf{S}+\mathbf{N}
$$

where $\mathbf{Y}$ is the noisy observation vector, $\mathbf{S}$ represents the transmitted signal for the alternative hypothesis $\left(H_{1}\right)$, and $\mathbf{N}$ is the noise component that is independent of $\mathbf{S}$. Instead of using a constant level for $\mathbf{S}$ as in the conventional case, one can consider a more generic scenario in which the signal $\mathbf{S}$ can be stochastic. Then, the aim is to find the optimal PDF for $\mathbf{S}$ in (1) and the corresponding decision rule that maximize the probability of detection under the constraints on the probability of false alarm and average transmit power. A feedback mechanism from the receiver to the transmitter is assumed to facilitate the joint optimization of the signaling structure and the decision rule, which is a reasonable assumption, for example, for cognitive radio (CR) systems.

Note that the probability distribution of the noise component in (1) is not necessarily Gaussian. Due to interference, such as multiple-access interference, the noise component can have a significantly different probability distribution from the 
Gaussian distribution [8].

At the receiver, the structure of a randomized test is assumed to choose between the two hypotheses. Such a test is completely characterized by a decision rule $\phi$. For a given observation vector $\mathbf{y}$, this test accepts hypothesis $H_{1}$ with probability $\phi(\mathbf{y})$, and rejects it with probability $1-\phi(\mathbf{y})$ where $0 \leq \phi(\mathbf{y}) \leq 1$ for all $\mathbf{y}$. If $\phi$ takes on only the values 0 and 1 , the test reduces to a nonrandomized one, and $\phi$ simply becomes the indicator function of the decision region [9].

In the NP framework, for a given value of $\alpha \in(0,1)$, the aim is to maximize the probability of detection such that the probability of false alarm does not exceed $\alpha$. In other words, the tradeoff between type-I and type-II errors is taken into account in the NP approach [7]. Given the decision rule (detector) $\phi$, the two probabilities of interest, the probability of detection $\mathrm{P}_{\mathrm{D}}$ and the probability of false alarm $\mathrm{P}_{\mathrm{FA}}$, can be calculated as follows:

$$
\begin{aligned}
\mathrm{P}_{\mathrm{D}} & =\mathbb{E}_{1}\{\phi(\mathbf{Y})\}=\int_{\mathbb{R}^{M}} \phi(\mathbf{y}) p_{1}(\mathbf{y}) \mathrm{d} \mathbf{y} \\
\mathrm{P}_{\mathrm{FA}} & =\mathbb{E}_{0}\{\phi(\mathbf{Y})\}=\int_{\mathbb{R}^{M}} \phi(\mathbf{y}) p_{0}(\mathbf{y}) \mathrm{d} \mathbf{y}
\end{aligned}
$$

where $p_{i}(\mathbf{y})$ denotes the conditional PDF of the observation when hypothesis $H_{i}$ is assumed to be true for $i \in\{0,1\}$, and the subscripts on the expectation operators indicate the corresponding hypotheses. Since stochastic signaling is considered, $\mathbf{S}$ in (1) is modeled as a random vector. Recalling that the signal and the noise are independent, the conditional PDF of the observation under the alternative hypothesis $H_{1}$ can be calculated as $p_{1}(\mathbf{y})=\int_{\mathbb{R}^{M}} p_{\mathbf{S}}(\mathbf{x}) p_{\mathbf{N}}(\mathbf{y}-\mathbf{x}) \mathrm{d} \mathbf{x}=$ $\mathbb{E}\left\{p_{\mathbf{N}}(\mathbf{y}-\mathbf{S})\right\}$, where the expectation is taken over the PDF of $\mathbf{S}$. On the other hand, the conditional PDF of the observation under the null hypothesis $H_{0}$ is given simply by $p_{0}(\mathbf{y})=p_{\mathbf{N}}(\mathbf{y})$. Then, using the linearity of the expectation operator, the probability of detection can be expressed as

$$
\begin{aligned}
\mathrm{P}_{\mathrm{D}} & =\int_{\mathbb{R}^{M}} \phi(\mathbf{y}) \mathbb{E}\left\{p_{\mathbf{N}}(\mathbf{y}-\mathbf{S})\right\} \mathrm{d} \mathbf{y} \\
& =\mathbb{E}\left\{\int_{\mathbb{R}^{M}} \phi(\mathbf{y}) p_{\mathbf{N}}(\mathbf{y}-\mathbf{S}) \mathrm{d} \mathbf{y}\right\} \triangleq \mathbb{E}\{h(\phi ; \mathbf{S})\}
\end{aligned}
$$

and the probability of false alarm $\mathrm{P}_{\mathrm{FA}}$ is given by

$$
\mathrm{P}_{\mathrm{FA}}=\int_{\mathbb{R}^{M}} \phi(\mathbf{y}) p_{\mathbf{N}}(\mathbf{y}) \mathrm{d} \mathbf{y} .
$$

In practical systems, there is a constraint on the average power emitted from the transmitter. Under the framework of stochastic signaling, this constraint on the average power can be expressed in the following form [7]:

$$
\mathbb{E}\left\{\|\mathbf{S}\|_{2}^{2}\right\} \leq \mathrm{A}
$$

where A denotes the average power limit.

One of the main motivations behind this study is to understand how stochastic signaling can help improve the detection performance of an on-off keying system without violating the constraint on the false alarm probability. Under the NP decision criterion, the optimal signaling and detector design problem can then be stated as

$$
\begin{aligned}
\max _{\left\{\phi, p_{\mathbf{S}}\right\}} & \mathbb{E}\{h(\phi ; \mathbf{S})\} \\
\text { subject to } & \mathrm{P}_{\mathrm{FA}} \leq \alpha \text { and } \mathbb{E}\left\{\|\mathbf{S}\|_{2}^{2}\right\} \leq \mathrm{A}
\end{aligned}
$$

where the expectations are taken over the PDF of S, $\alpha \in$ $(0,1)$, and $h(\phi ; \mathbf{S})$ and $\mathrm{P}_{\mathrm{FA}}$ are as in (3) and (4), respectively. Note that there are also implicit constraints in the optimiza- tion problem in (6), since $p_{\mathbf{S}}(\cdot)$ represents a PDF. Namely, $p_{\mathbf{S}}(\mathbf{x}) \geq 0, \forall \mathbf{x} \in \mathbb{R}^{M}$, and $\int_{\mathbb{R}^{M}} p_{\mathbf{S}}(\mathbf{x}) \mathrm{d} \mathbf{x}=1$ should also be satisfied by the optimal solution.

Although the optimization problem in (6) provides a generic formulation that is valid for any noise PDF, it is difficult to solve in general as the optimization needs to be performed over a space of signal PDFs and decision rules. In the following analysis, it is proven that optimizing over a set of variables (instead of functions) is sufficient to obtain the optimal signal PDF and the decision rule. To that aim, the following lemma is presented first.

Lemma 1: Given a decision rule $\phi$ that satisfies the false alarm constraint; if $h(\phi ; \mathbf{s})$ in (3) is a continuous function of $\mathbf{s}$ defined on a compact subset of $\mathbb{R}^{M}$, then an optimal solution to (6) can be expressed in the form of

$$
p_{\mathbf{S}}^{\mathrm{opt}}(\mathbf{x})=\lambda \delta\left(\mathbf{x}-\mathbf{s}_{1}\right)+(1-\lambda) \delta\left(\mathbf{x}-\mathbf{s}_{2}\right)
$$

where $\lambda \in[0,1]$.

Proof: Suppose that a decision rule $\tilde{\phi}$ is given such that the constraint on the probability of false alarm is satisfied, i.e., $\int_{\mathbb{R}^{M}} \tilde{\phi}(\mathbf{y}) p_{\mathbf{N}}(\mathbf{y}) \mathrm{d} \mathbf{y} \leq \alpha$. Then, $h(\tilde{\phi} ; \mathbf{s})=$ $\int_{\mathbb{R}^{M}} \tilde{\phi}(\mathbf{y}) p_{\mathbf{N}}(\mathbf{y}-\mathbf{s}) \mathrm{d} \mathbf{y}$ in (3) becomes a function of $\mathbf{s}$ only. Formally, $\mathrm{P}_{\mathrm{D}}$ in (3) can be represented as $\mathrm{P}_{\mathrm{D}}=\mathbb{E}\{h(\mathbf{S})\}$ and the optimization problem in (6) can be stated as

$$
\max _{p_{\mathbf{S}}} \mathbb{E}\{h(\mathbf{S})\} \quad \text { subject to } \quad \mathbb{E}\left\{\|\mathbf{S}\|_{2}^{2}\right\} \leq \mathrm{A} .
$$

Similar optimization problems have been studied extensively in the literature under various frameworks $[1,2,5,10]$. Given the conditions in the lemma, Carathéodory's theorem from convex analysis [11] implies that the optimal solution of (8) can be expressed by a randomization of at most two signal vectors. Therefore, for any decision rule $\tilde{\phi}$ satisfying the false alarm constraint, the optimal signal PDF can be represented as in (7).

At this point, it should be emphasized that the above lemma points out to a significant reduction on the complexity of the optimization problem under certain conditions. Namely, the optimal signal design no longer involves a search over all possible signal PDFs; but instead a randomization between at most two different signal vectors suffices. Hence, the problem in (6) can be solved over the signal PDFs that are in the form of (7). Led by this observation, a further simplification of the optimization problem is presented.

Proposition 1: Under the conditions in Lemma 1, the optimization problem in (6) can be expressed as follows:

$$
\begin{aligned}
\max _{\left\{\lambda, \mathbf{s}_{1}, \mathbf{s}_{2}, \eta\right\}} & \int_{\Gamma}\left\{\lambda p_{\mathbf{N}}\left(\mathbf{y}-\mathbf{s}_{1}\right)+(1-\lambda) p_{\mathbf{N}}\left(\mathbf{y}-\mathbf{s}_{2}\right)\right\} \mathrm{d} \mathbf{y} \\
\text { subject to } & \int_{\Gamma} p_{\mathbf{N}}(\mathbf{y}) \mathrm{d} \mathbf{y}=\alpha \\
& \lambda\left\|\mathbf{s}_{1}\right\|_{2}^{2}+(1-\lambda)\left\|\mathbf{s}_{2}\right\|_{2}^{2} \leq \mathrm{A} \\
& \lambda \in[0,1] \text { and } \eta \geq 0
\end{aligned}
$$

where $\Gamma=\left\{\mathbf{y} \in \mathbb{R}^{M}: \lambda p_{\mathbf{N}}\left(\mathbf{y}-\mathbf{s}_{1}\right)+(1-\lambda) p_{\mathbf{N}}\left(\mathbf{y}-\mathbf{s}_{2}\right)\right.$ $\left.>\eta p_{\mathbf{N}}(\mathbf{y})\right\}$, and $\alpha \in(0,1)$.

Proof: It is known that the NP detector gives the most powerful $\alpha$-level test of $H_{0}$ versus $H_{1}$ [7]. In other words, when the aim is to maximize the probability of detection such that the probability of false alarm does not exceed a predetermined value $\alpha$, the NP detector is the optimal choice. When deciding between two simple hypotheses $H_{0}$ versus $H_{1}$ based on observation $\mathbf{y}$, the NP decision rule takes the following 
form of an LRT:

$$
\tilde{\phi}_{N P}(\mathbf{y})= \begin{cases}1, & \text { if } p_{1}(\mathbf{y})>\eta p_{0}(\mathbf{y}) \\ \gamma(\mathbf{y}), & \text { if } p_{1}(\mathbf{y})=\eta p_{0}(\mathbf{y}) \\ 0, & \text { if } p_{1}(\mathbf{y})<\eta p_{0}(\mathbf{y})\end{cases}
$$

where $\eta \geq 0$ and $0 \leq \gamma(\mathbf{y}) \leq 1$ are chosen such that the probability of false alarm satisfies $\mathrm{P}_{\mathrm{FA}}=\mathbb{E}_{0}\left\{\tilde{\phi}_{N P}(\mathbf{Y})\right\}=\alpha$, where the expectation is taken with respect to the null hypothesis $H_{0}$. Then, the NP decision rule is the optimal one among all $\alpha$-level decision rules, i.e. $\mathrm{P}_{\mathrm{D}}=\mathbb{E}_{1}\left\{\tilde{\phi}_{N P}(\mathbf{Y})\right\}$ is maximized, where the expectation is taken with respect to the alternative hypothesis $H_{1}$. It can be proven that such a rule always exists for all $\alpha \in(0,1)$ and is unique [7]. Let $L(\mathbf{Y})=p_{1}(\mathbf{Y}) / p_{0}(\mathbf{Y})$ be the likelihood function. For continuous $L(\mathbf{Y}), \gamma(\mathbf{Y})$ can be chosen arbitrarily since the probability of the event $\left\{p_{1}(\mathbf{Y})=\eta p_{0}(\mathbf{Y})\right\}$ is equal to 0 under both $H_{0}$ and $H_{1}[7,9]$.

To keep the formulation simpler, the PDF of the channel noise is assumed to be continuous which gives rise to a continuous likelihood function. An extension for the discrete case is straightforward by incorporating the parameter $\gamma(\mathbf{y})$ into the calculations for the detection and false alarm probabilities. Under the continuity assumption, while deciding between two simple hypotheses based on observation $\mathbf{y}$, the NP decision rule, which selects hypothesis $H_{1}$ if $p_{1}(\mathbf{y})>\eta p_{0}(\mathbf{y})$ and selects hypothesis $H_{0}$ otherwise, maximizes the probability of detection under the false alarm constraint. Therefore, when the signal PDF $p_{\mathbf{S}}(\cdot)$ is specified, it is sufficient to consider only the detection probability of the NP rule instead of a search over all the decision rules.

As the NP decision rule assigns observation y to hypothesis $H_{1}$ if $p_{1}(\mathbf{y})>\eta p_{0}(\mathbf{y})$ and decides hypothesis $H_{0}$ otherwise, the probability of detection and false alarm expressions in (2) can be expressed for an NP decision rule as $\mathrm{P}_{\mathrm{D}}=$ $\mathbb{E}_{1}\left\{\tilde{\phi}_{N P}(\mathbf{Y})\right\}=\int_{\Gamma} p_{1}(\mathbf{y}) \mathrm{d} \mathbf{y}$ and $\mathrm{P}_{\mathrm{FA}}=\mathbb{E}_{0}\left\{\tilde{\phi}_{N P}(\mathbf{Y})\right\}=$ $\int_{\Gamma} p_{0}(\mathbf{y}) \mathrm{d} \mathbf{y}$, where $\Gamma=\left\{\mathbf{y} \in \mathbb{R}^{M}: p_{1}(\mathbf{y})>\eta p_{0}(\mathbf{y})\right\}$.

In Lemma 1, it is shown that an optimal signal PDF is in the form of (7). As a result, the conditional PDF of the observation under hypothesis $H_{1}$ can be written as $p_{1}(\mathbf{y})=\mathbb{E}\left\{p_{\mathbf{N}}(\mathbf{y}-\mathbf{S})\right\}=\int_{\mathbb{R}^{M}} p_{\mathbf{S}}(\mathbf{x}) p_{\mathbf{N}}(\mathbf{y}-\mathbf{x}) \mathrm{d} \mathbf{x}=$ $\lambda p_{\mathbf{N}}\left(\mathbf{y}-\mathbf{s}_{1}\right)+(1-\lambda) p_{\mathbf{N}}\left(\mathbf{y}-\mathbf{s}_{2}\right)$. Similarly, the average power constraint in (6) becomes $\lambda\left\|\mathbf{s}_{1}\right\|_{2}^{2}+(1-\lambda)\left\|\mathbf{s}_{2}\right\|_{2}^{2} \leq$ A. Therefore, the expressions for $\mathrm{P}_{\mathrm{D}}$ and $\mathrm{P}_{\mathrm{FA}}$ at the end of the previous paragraph imply that the optimization problems in (6) and (9) are equivalent as stated in the proposition.

Proposition 1 implies that the solution of the original optimization problem in (6), which considers the joint optimization of the stochastic signal PDF and the detector, can be obtained as the solution of the much simpler optimization problem specified in (9).

Comparing the formulations in (6) and (9), it is noted that a significant complexity reduction is obtained in the representation of the problem by optimizing over a set of variables instead of a set of functions. The solution of the optimization problem in (9) can be obtained via global optimization techniques (since it is not a convex problem in general), or a convex relaxation approach as in [12] can be employed to obtain approximate solutions in polynomial time. In this study, the multistart and patternsearch methods from MATLAB's Global Optimization Toolbox are used to obtain the solution of (9).

Assuming that the selected optimization algorithm successfully returns the parameters $\left\{\lambda^{\mathrm{opt}}, \mathbf{s}_{1}^{\mathrm{opt}}, \mathbf{s}_{2}^{\mathrm{opt}}, \eta^{\mathrm{opt}}\right\}$ for the problem in (9), the optimal signal PDF can be constructed as $p_{\mathbf{S}}^{\mathrm{opt}}(\mathbf{x})=\lambda^{\mathrm{opt}} \delta\left(\mathbf{x}-\mathbf{s}_{1}^{\mathrm{opt}}\right)+\left(1-\lambda^{\mathrm{opt}}\right) \delta\left(\mathbf{x}-\mathbf{s}_{2}^{\mathrm{opt}}\right)$ and the optimal decision rule assumes the form of the corresponding NP decision rule that decides hypothesis $H_{1}$ if $\lambda^{\mathrm{opt}} p_{\mathbf{N}}\left(\mathbf{y}-\mathbf{s}_{1}^{\mathrm{opt}}\right)+\left(1-\lambda^{\mathrm{opt}}\right) p_{\mathbf{N}}\left(\mathbf{y}-\mathbf{s}_{2}^{\mathrm{opt}}\right)>\eta^{\mathrm{opt}} p_{\mathbf{N}}(\mathbf{y})$ and decides hypothesis $H_{0}$ otherwise.

\section{SIMULATION RESULTS AND CONCLUSIONS}

In this section, the optimal signal PDF and optimal detector parameters are obtained by applying the theory developed in the previous section along with the results from two closely related signaling techniques. A binary hypothesestesting problem specified as in (1) is considered with scalar observations. Such a scenario is well suited for binary communications systems that transmit no signal for bit 0 and a signal (or a randomization of two signal values as discussed above) for bit 1 (i.e., on-off keying). The noise $N$ in (1) is modeled as a symmetric Gaussian mixture noise with equal variances, the PDF of which can be expressed as $p_{N}(n)=\sum_{i=1}^{L} l_{i} \exp \left\{-\left(n-\mu_{i}\right)^{2} /\left(2 \sigma^{2}\right)\right\} /(\sqrt{2 \pi} \sigma)$ [4]. It is noted that the average power of the noise can be calculated from $\mathbb{E}\left\{N^{2}\right\}=\sigma^{2}+\sum_{i=1}^{L} l_{i} \mu_{i}^{2}$. Similar to those introduced in [2], three different signaling schemes are considered.

Conventional Solution: In this case, the transmitter employs deterministic signaling at the maximum permitted power level, which is known to be optimal if the noise present in the channel were Gaussian distributed. To mitigate the effects of non-Gaussian channel noise, the receiver is assumed to know the channel statistics and allowed to design the optimal NP decision rule corresponding to the deterministic signaling at the power limit. This optimization problem can be expressed as follows:

$$
\max _{\eta} \int_{\Gamma} p_{N}(y-A) \mathrm{d} y \text { s.t. } \int_{\Gamma} p_{N}(y) \mathrm{d} y=\alpha, \eta \geq 0
$$

where $\Gamma=\left\{y \in \mathbb{R}: p_{N}(y-A)>\eta p_{N}(y)\right\}$ and $\alpha \in(0,1)$.

Optimal-Stochastic: This approach refers to the joint design of the signaling structure and the decision rule formulated in (6), which can also be obtained from (9) as studied in the previous section.

Optimal-Deterministic: A simplified version of the optimal solution in (9) can be obtained by assuming that the transmitted signal is deterministic; i.e., it is not a randomization of two distinct signal levels. The optimization problem in (9) reduces to

$$
\begin{aligned}
& \max _{\{s, \eta\}} \int_{\Gamma} p_{N}(y-s) \mathrm{d} y \\
& \text { subject to } \int_{\Gamma} p_{N}(y) \mathrm{d} y=\alpha,|s|^{2} \leq \mathrm{A}, \quad \eta \geq 0
\end{aligned}
$$

where $\Gamma=\left\{y \in \mathbb{R}: p_{N}(y-s)>\eta p_{N}(y)\right\}$ and $\alpha \in(0,1)$.

In Fig. 1, the detection probabilities of the schemes described above are plotted versus $\sigma$ for $\mathrm{A}=1$ and $\alpha=0.05$, where the parameters of the Gaussian mixture noise are given

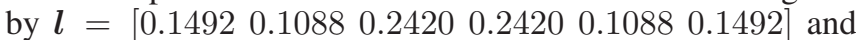
$\boldsymbol{\mu}=[-1.211-0.755-0.30 .30 .7551 .211]^{2}$ From the figure, it is observed that the conventional solution has the worst performance as expected since no optimization is performed

${ }^{2}$ In obtaining the optimal solutions for the global optimization problems stated above, MATLAB's multistart method is employed with 250 random start points and $s q p$ algorithm is used together with the local solver fmincon. The extrema returned by the method are cross-checked with the results from the patternsearch method. This procedure is repeated for all values of $\sigma$ in the set $\{0.01: 0.005: 0.50\}$. 


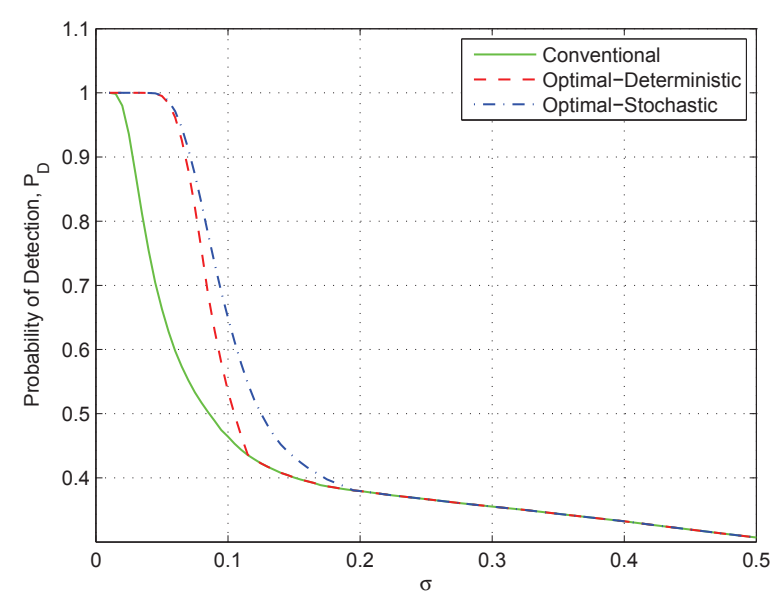

Fig. 1. Probability of detection $\mathrm{P}_{\mathrm{D}}$ versus $\sigma$ for different approaches when $\mathrm{A}=1$ and $\alpha=0.05$.

for the signaling scheme employed at the transmitter. As mentioned above, signaling at the maximum permitted power level is not necessarily optimal for non-Gaussian cases. Having a multimodal PDF, channel noise degrades the performance of the communications system when the on-signal (symbol 1) is transmitted at the power limit. Optimizing deterministic signal levels improves over the performance of the conventional solution for $0.01 \leq \sigma \leq 0.115$ as observed from the OptimalDeterministic curve by avoiding the overlaps among the components of the Gaussian mixture noise more effectively. Further performance improvements are obtained over a larger interval $0.01<\sigma<0.20$ when optimal stochastic signals are considered instead of conventional signaling (see OptimalStochastic). The superior performance of optimal stochastic signaling over optimal deterministic signaling is also evident from the values assumed by the probability of detection curves for $0.04 \leq \sigma \leq 0.20$. In contrast to finding the single signal value that best avoids the overlaps among mixture components, stochastic signaling scheme allots the available power in such a way that a large portion of the power is allocated to the signal component that results in less overlap between the original and the shifted noise PDF on average. Hence, the best probability of detection performance is achieved by the solution of the joint optimization problem investigated in the previous section, which performs a randomization between two signal values for symbol 1 , and employs the corresponding $\alpha$-level NP decision rule at the receiver. For example, at $\sigma=0.1$, the optimal stochastic signal is a randomization of $s_{1}=0.2732$ and $s_{2}=1.2460$ with $\lambda=0.3739$, achieving a detection probability of 0.6494 . On the other hand, the optimal deterministic solution sets $s=0.7684$, resulting in a detection probability of 0.5345 .

As $\sigma$ is increased beyond 0.20 , it is observed that both optimal signaling schemes converge to conventional signaling. This is mainly due to the fact that the overlap among mixture components of the noise PDF becomes significant for large values of $\sigma$, and there is not enough freedom left for the randomization to become effective over transmitting at the power limit. It is also concluded from the results of the previous section that the performance figure achieved via randomization is the global optimum; that is, it cannot be beaten by the combination of any different signaling schemes with a single detector as long as the problem formulation stays the same. In order to explain the results depicted in Fig. 1, Table 1 presents the solutions of the optimization problems in (11), (12), and (9) for the Conventional, Optimal-Deterministic and OptimalStochastic approaches, respectively. It is observed that Table 1 is in agreement with Fig. 1.

\begin{tabular}{|c|c|cc|cccc|}
\hline & Conv. & \multicolumn{2}{|c|}{ Deterministic } & \multicolumn{4}{|c|}{ Stochastic } \\
$\sigma$ & $\eta$ & $S$ & $\eta$ & $\lambda$ & $S_{1}$ & $S_{2}$ & $\eta$ \\
\hline \hline 0.010 & 0.0001 & 0.291 & 2.395 & 1 & 0.294 & N/A & 3.936 \\
0.050 & 3.021 & 0.240 & 0.134 & 0.313 & 1.751 & 0.241 & 0.112 \\
0.075 & 3.034 & 0.251 & 2.708 & 0.386 & 0.251 & 1.261 & 1.890 \\
0.100 & 2.870 & 0.768 & 3.639 & 0.374 & 0.273 & 1.246 & 3.009 \\
0.125 & 2.540 & 1 & 2.540 & 0.518 & 1.227 & 0.677 & 2.779 \\
0.150 & 2.236 & 1 & 2.236 & 0.455 & 0.640 & 1.222 & 2.315 \\
0.175 & 1.989 & 1 & 1.989 & 0.390 & 0.601 & 1.187 & 2.112 \\
0.200 & 1.822 & 1 & 1.822 & 0.333 & 1.001 & 0.9996 & 1.822 \\
0.250 & 1.931 & 1 & 1.931 & 1 & 1 & N/A & 1.931 \\
0.300 & 2.071 & 1 & 2.071 & 1 & 1 & N/A & 2.071 \\
0.350 & 2.216 & 1 & 2.216 & 1 & 1 & N/A & 2.216 \\
0.400 & 2.348 & 1 & 2.348 & 1 & 1 & N/A & 2.348 \\
0.450 & 2.473 & 1 & 2.473 & 1 & 1 & N/A & 2.473 \\
0.500 & 2.569 & 1 & 2.569 & 1 & 1 & N/A & 2.569 \\
\hline
\end{tabular}

Table 1. Conventional, Optimal-Deterministic, and OptimalStochastic Signaling

\section{REFERENCES}

[1] C. Goken, S. Gezici, and O. Arikan, "Optimal stochastic signaling for power-constrained binary communications systems," IEEE Trans. Wireless Commun., vol. 9, no. 12, pp. 3650-3661, Dec. 2010.

[2] C. Goken, S. Gezici, and O. Arikan, "Optimal signaling and detector design for power-constrained binary communications systems over non-Gaussian channels," IEEE Commun. Lett., vol. 14, pp. 100-102, Feb. 2010.

[3] M. Azizoglu, "Convexity properties in binary detection problems," IEEE Trans. Inform. Theory, vol. 42, no. 4, pp. 1316-1321, July 1996.

[4] V. Bhatia and B. Mulgrew, "Non-parametric likelihood based channel estimator for Gaussian mixture noise," Signal Processing, vol. 87, pp. 2569-2586, Nov. 2007.

[5] A. Patel and B. Kosko, "Optimal noise benefits in Neyman-Pearson and inequality-constrained signal detection," IEEE Trans. Sig. Processing, vol. 57, no. 5, pp. 1655-1669, May 2009.

[6] I. Korn, J. P. Fonseka, and S. Xing, "Optimal binary communication with nonequal probabilities," IEEE Trans. Commun., vol. 51, pp. 1435-1438, Sep. 2003.

[7] H. V. Poor, An Introduction to Signal Detection and Estimation, Springer-Verlag, New York, 1994.

[8] T. Erseghe, V. Cellini, and G. Dona, "On UWB impulse radio receivers derived by modeling MAI as a Gaussian mixture process," IEEE Trans. Wireless Commun., vol. 7, no. 6, pp. 2388-2396, June 2008.

[9] E. L. Lehmann, Testing Statistical Hypotheses, Chapman \& Hall, New York, 2 edition, 1986.

[10] H. Chen, P. K. Varshney, S. M. Kay, and J. H. Michels, "Theory of the stochastic resonance effect in signal detection: Part I-Fixed detectors," IEEE Trans. Sig. Processing, vol. 55, no. 7, pp. 3172-3184, July 2007.

[11] R. T. Rockafellar, Convex Analysis, Princeton University Press, Princeton, NJ, 1968.

[12] S. Bayram, S. Gezici, and H. V. Poor, "Noise enhanced hypothesis-testing in the restricted Bayesian framework," IEEE Trans. Sig. Processing, vol. 58, no. 8, pp. 3972-3989, Aug. 2010. 